\title{
Pelargonium radens Oil
}

National Cancer Institute

\section{Source}

National Cancer Institute. Pelargonium radens Oil. NCI Thesaurus. Code C107345.

The essential oil of Pelargonium radens. Pelargonium radens oil is used for its aromatic properties and as an ing redient in skincare preparations. 